\section{International Scientific Journal Theoretical \& Applied Science}

p-ISSN: 2308-4944 (print)

e-ISSN: 2409-0085 (online)

Year: 2016

Issue: 12

Published: 30.12 .2016

http://T-Science.org

SECTION 9. Chemistry and chemical technology.
Sabir Ahmad Mammadov

Doctor in Chemistry, Professor, Head of Laboratory, Institute of Chemistry of Additives,

Azerbaijan National Academy of Sciences,

Azerbaijan

sabir.mamedov.39@mail.ru

Sevgili Ismayil Mammadova

$\mathrm{PhD}$ in Chemistry, doctorant, Institute of Chemistry of Additives,

Azerbaijan National Academy of Sciences,

Azerbaijan

alximikseva@rambler.ru

Nina Petrovna Ladokhina

$\mathrm{PhD}$ in Chemistry, Assistant professor,

Leadinq Scientific Researcher,

Institute of Chemistry of Additives,

Azerbaijan National Academy of Sciences,

Azerbaijan

$\underline{\text { nina62_62@mail.ru }}$

Isa Shahruddin Huseinov

$\mathrm{PhD}$ in Chemistry,

Leading Scientific Researcher,

Institute of Chemistry of Additives,

Azerbaijan National Academy of Sciences,

Azerbaijan

Shefa Kazim Kazimzade

aspirant,

Institute of Chemistry of Additives,

Azerbaijan National Academy of Sciences,

Azerbaijan

\title{
STUDY OF REACTION OF ARYLSULFOCHLORIDES WITH BICYCLIC AMINES
}

Abstract: The reaction of arylsulfochlorides with 6-(adamantyl-1)-2-z-3-(4-arylsulfonyl)-[2, 3-b] pyridineselenophen- or thiophen was studied. It was found that regardless of the nature of functional groups in 2position the output of heterosulfamides with selenophens is lower than with thiophen fragment. The effort to obtain sulfamides with bicyclic amines containing nitrile group in 2-position, failed. Some adamantyl-pyridineselenophens and - thiophens were tested as a bactericide against staphylococcus and typhoid fever. It was found that selenium containing products are more effective than sulfur-containing compounds.

Key words: heterosulfamides, aminoheterocycles, bicyclic amines, polarophil, heterocyclization, bactericide

Language: English

Citation: Mammadov SA, Mammadova SI, Ladokhina NP, Huseinov IS, Kazimzade SK (2016) STUDY OF REACTION OF ARYLSULFOCHLORIDES WITH BICYCLIC AMINES. ISJ Theoretical \& Applied Science, 12 (44): 94-97.

Soi: http://s-o-i.org/1.1/TAS-12-44-19 Doi: crossef http://dx.doi.org/10.15863/TAS.2016.12.44.19

\section{INTRODUCTION}

Medications improving brain blood circulationnimodipine, nifedimin-(blockers of calcium channels) were produced on the basis of pyridinethion compounds. At present active searches of non-glycoside and nonadrenergic cardiotonic agents with large therapeutic are conducted. Their synthetic analogs - acrinon, proximone, milrinone are widely applied in intensive therapy. Besides, sulphamides containing pyrimidine fragments have cytostatic action that allows using them as antiviral and antineoplastic medicines. They are potential bactericides. Their antimicrobial activity and influence on various microorganisms depends by

ISPC Generalization of scientific results, 
nature of heterocycle and functional groups. Therefore, the synthesis of new sulphamides containing bicyclic compounds, by reaction of arylsulfochlorides with heterocyclic amines is urgent.

The influence of the composition of heterocycle and position of amino groups was studied. So, the location of amino groups in isoxazole strongly influences on its reactivity. During the reaction of sulfochlorides with 5-amino 3,4 dimethyl isoxazole [1] and biphenylisoxazole [2] we obtained hetarylsulfamides with high yield. Reaction of sulfochlorides with five-membered aminoheterocycles, such as oxazoles [3], pyrazole proceeds with high yields. However, the reaction of sulfochlorides with benzoxazole requires long boiling in solution of pyridine [4].

Reaction of arylsulfochlorides with piperazines, attached to them through oxygen or $\mathrm{N}$ pyridylil- or pyrimidinediyle fragment, proceeds very easily. Action of radicals and functional groups on reaction wasn't observed [5]. Obtained sulphamides can be applied at diseases of CNS and decreased kidney function.

Despite contents of cinchine acid in 4aminobenzenesulfochloride fragment, the reaction with a 2-amino-4,6-dimethylpyrimidine proceeds
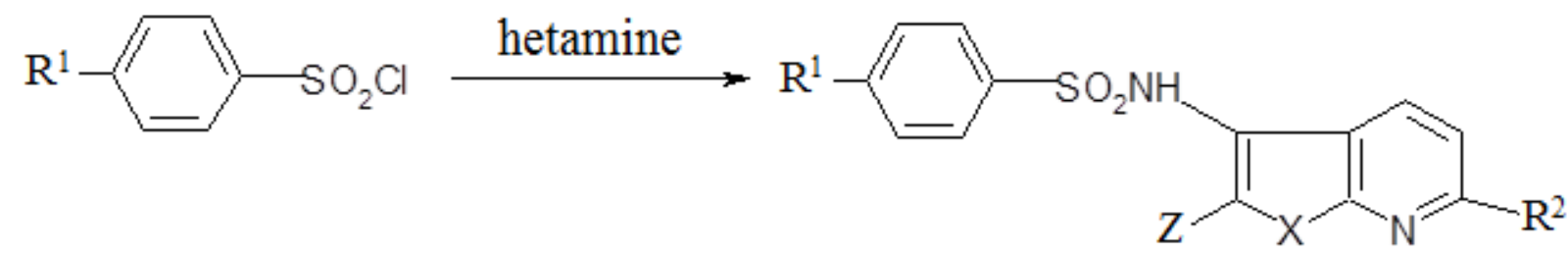

$$
\begin{array}{r}
\mathrm{R}^{1}=\mathrm{H}, \mathrm{R}^{2}=\mathrm{Ad}_{1}: \mathrm{Z}=\mathrm{COOC}_{2} \mathrm{H}_{5}, \mathrm{X}=\mathrm{S}(\mathrm{I}) ; \\
\mathrm{Z}=\mathrm{COOC}_{6} \mathrm{H}_{5}, \mathrm{X}=\mathrm{Se}(\mathrm{II}) ; \mathrm{Z}=\mathrm{COOH}_{,} \mathrm{X}=\mathrm{Se}(\mathrm{III}) ; \\
\mathrm{Z}=\mathrm{COC}_{6} \mathrm{H}_{5}, \mathrm{X}=\mathrm{S}(\mathrm{IV}) ; \\
\mathrm{R}^{1}=\mathrm{CH}_{3}, \mathrm{R}^{2}=\mathrm{Ad}_{1}, \mathrm{Z}=\mathrm{COOC}_{2} \mathrm{H}_{5}, \mathrm{X}=\mathrm{S}(\mathrm{V}) ; \\
\mathrm{R}^{1}=\mathrm{CH}_{3}, \mathrm{R}^{2}=\mathrm{Ad}_{1}, \mathrm{Z}=\mathrm{CONH}_{2}, \mathrm{X}=\mathrm{Se}(\mathrm{VI}) ; \\
\mathrm{R}^{1}=\mathrm{CH}_{3}, \mathrm{R}^{2}=\mathrm{CH}_{3}, \mathrm{Z}=\mathrm{COOC}_{2} \mathrm{H}_{5}, \mathrm{X}=\mathrm{S}(\mathrm{VII}) ; \\
\mathrm{R}^{1}=\mathrm{H}, \mathrm{R}^{2}=\mathrm{CH}_{3}, \mathrm{Z}=\mathrm{COOC}_{2} \mathrm{H}_{5}, \mathrm{X}=\mathrm{S}(\mathrm{VIII})
\end{array}
$$

It is found that in the presence of selenium atom, regardless of functional groups in 2-position, the yields of hetarylsulfamide are lower, than in sulfur atom. Besides, it should be noted that the content of such voluminous fragment like adamantyl1 influences on yields of compounds.

Selective testing of some hetarylsulfamides (II, IV, V, VI, VII) as bactericides against staphylococcus and typhoid fever agents (S.typhi) was conducted. Obtained data are provided in table 1. As table 1 shows hetarylsulfamides containing selenium (compounds II, VI), regardless of the nature of functional groups $\left(\mathrm{COC}_{6} \mathrm{H}_{5}, \mathrm{CONH}_{2}\right)$ are more effective, than sulfur-containing sulphamides. They even in concentration of $0,01 \%$ in solution of $45 \%$ of ethanol within 60 min. completely destroy microorganisms of staphylococcus and stop the development of S.typhi. In comparison with compound II, sulfur-containing compound IV affects only in concentration in $0,05 \%$ for $30 \mathrm{~min}$. The same is observed with compounds V and VII. Full elimination of staphylococcus happens in concentration of $0,1 \%$ for $60 \mathrm{~min}$., and for S.typhi the elimination occurs in concentration of $0,05 \%$ for 30 min. In absence of adamantine fragment (compound VII) in heterocycle bactericidal action weakens.

Long latent period can be explained with large size of hetarylsulfamides and the reason of difficult penetration through membrane of microorganism. 


\begin{tabular}{|c|c|c|c|c|c|c|}
\hline Impact Factor: & $\begin{array}{l}\text { ISRA (India) } \\
\text { ISI (Dubai, UAF } \\
\text { GIF (Australia) } \\
\text { JIF }\end{array}$ & $\begin{array}{l}=1.344 \\
=0.829 \\
=0.564 \\
=1.500\end{array}$ & $\begin{array}{l}\text { SIS (USA) } \\
\text { PИНЦ (Russia) } \\
\text { ESJI (KZ) } \\
\text { SJIF (Morocco) }\end{array}$ & $\begin{array}{l}=0.912 \\
=0.234 \\
=1.042 \\
=2.031\end{array}$ & $\begin{array}{l}\text { ICV (Poland) } \\
\text { PIF (India) } \\
\text { IBI (India) }\end{array}$ & $\begin{array}{l}=6.630 \\
=1.940 \\
=4.260\end{array}$ \\
\hline
\end{tabular}

Table 1

\begin{tabular}{|c|c|c|c|c|c|c|c|c|}
\hline \multirow{3}{*}{$\begin{array}{l}\text { Strains } \\
\text { cultures }\end{array}$} & \multicolumn{8}{|c|}{ Concentration, $\%$} \\
\hline & \multicolumn{4}{|c|}{$0,01 \%$} & \multicolumn{4}{|c|}{$0,05 \%$} \\
\hline & 20 & 30 & 40 & 60 & 20 & 30 & 40 & 60 \\
\hline 1 & 2 & 3 & 4 & 5 & 6 & 7 & 8 & 9 \\
\hline & \multicolumn{8}{|c|}{$45 \%$ of alcohol solution in water } \\
\hline staphylococcus & + & + & + & + & & & & \\
\hline s. typhi & + & + & + & + & & & & \\
\hline & \multicolumn{8}{|c|}{ Compound II } \\
\hline staphylococcus & + & $\mathrm{x}$ & $\mathrm{x}$ & - & - & - & - & - \\
\hline s. typhi & + & + & + & $\mathrm{X}$ & $\mathrm{x}$ & - & - & - \\
\hline & \multicolumn{8}{|c|}{ Compound IV } \\
\hline staphylococcus & + & + & + & $\mathrm{X}$ & $\mathrm{x}$ & - & - & - \\
\hline s. typhi & + & + & + & + & + & $\mathrm{x}$ & - & - \\
\hline & \multicolumn{8}{|c|}{ Compound V } \\
\hline staphylococcus & + & + & + & $\mathrm{X}$ & - & - & - & - \\
\hline s. typhi & + & + & + & + & $\mathrm{x}$ & $\mathrm{x}$ & - & - \\
\hline & \multicolumn{8}{|c|}{ Compound VI } \\
\hline staphylococcus & + & + & $\mathrm{x}$ & - & - & - & - & - \\
\hline s. typhi & + & + & + & $\mathrm{x}$ & $\mathrm{X}$ & - & - & - \\
\hline
\end{tabular}

\section{EXPERIMENTAL PART}

PMR-spectra of some synthesized compounds were registered on a sepctrophotometer "Bruker" with operating frequency $90 \mathrm{MHz}$, IR spectra were registered on "Nicolet-is-10".

The synthesis of 6-(adamantyl-1) - 2-Z-3-3 (4-arylsulfonyl) - pyridine [2,3b] selenophen or thiophen.

General technique. $10 \mathrm{mmol}$ of the appropriate heterocyclic amine was dissolved in $20-25 \mathrm{ml}$ of freshly distilled pyridine and $11 \mathrm{mmol}$ of arylsulfochloride was slowly added to solution. The mixture was heated in case of $50-60^{\circ} \mathrm{C} 5-6$ hours, cooled and diluted with water before drop-out of crystals, filtered, washed out 3-4 times with water, dried and recrystallized from ethanol. Physical and chemical characteristics are provided in table 2.

PMR-and IR - spectral data are given in table 3, which confirm the supposed structures.

Table 2

Physical and chemical characteristics of hetarylsulfamides:

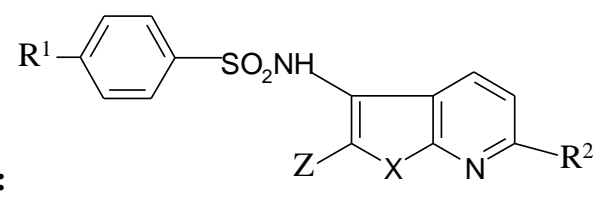

\begin{tabular}{|c|c|c|c|c|c|c|c|c|c|c|c|}
\hline & Z & $\mathrm{R}^{1}$ & $\mathrm{R}^{2}$ & $X$ & $\begin{array}{l}\text { Yield, } \\
\%\end{array}$ & $\begin{array}{l}\text { Tmelt. } \\
{ }^{0} \mathrm{C}\end{array}$ & $\begin{array}{l}\text { Chemical } \\
\text { formula }\end{array}$ & \multicolumn{4}{|c|}{ Analysis, \% } \\
\hline & & & & & & & & $\mathrm{C}$ & $\mathrm{H}$ & $\mathrm{N}$ & $S$ \\
\hline & & & & & & & & \multicolumn{4}{|c|}{$\begin{array}{c}\text { Found } \\
\text { Calculated, \% }\end{array}$} \\
\hline 1 & 2 & 3 & 4 & 5 & 6 & 7 & 8 & 9 & 10 & 11 & 12 \\
\hline I & $\mathrm{COOC}_{2} \mathrm{H}_{5}$ & $\mathrm{H}$ & $\mathrm{Ad}_{1}$ & S & 96,3 & $239-240.5$ & $\mathrm{C}_{26} \mathrm{H}_{27} \mathrm{~N}_{2} \mathrm{O}_{4} \mathrm{~S}_{2}$ & $\frac{63.29}{63.01}$ & $\frac{5.71}{5.49}$ & $\frac{5.39}{5.65}$ & $\frac{12.68}{12.90}$ \\
\hline II & $\mathrm{COC}_{6} \mathrm{H}_{5}$ & $\mathrm{H}$ & $\operatorname{Ad}_{1}$ & $\mathrm{Se}$ & 74,9 & 178-179 & $\mathrm{C}_{30} \mathrm{H}_{27} \mathrm{~N}_{2} \mathrm{O}_{3} \mathrm{SSe}$ & $\frac{62.98}{62.71}$ & $\frac{4.89}{4.74}$ & $\frac{4.65}{4.87}$ & --- \\
\hline III & $\mathrm{COOH}$ & $\mathrm{H}$ & $\mathrm{Ad}_{1}$ & $\mathrm{Se}$ & 68,6 & $\begin{array}{l}179.5- \\
181.5\end{array}$ & $\mathrm{C}_{24} \mathrm{H}_{23} \mathrm{~N}_{2} \mathrm{O}_{4} \mathrm{SSe}$ & $\frac{55.29}{56.03}$ & $\frac{4.68}{4.51}$ & $\frac{5.37}{5.45}$ & --- \\
\hline IV & $\mathrm{COC}_{6} \mathrm{H}_{5}$ & $\mathrm{H}$ & $\mathrm{Ad}_{1}$ & $S$ & 85,2 & $\begin{array}{l}170- \\
171.5\end{array}$ & $\mathrm{C}_{30} \mathrm{H}_{27} \mathrm{~N}_{2} \mathrm{O}_{3} \mathrm{~S}_{2}$ & $\frac{68.02}{68.29}$ & $\frac{5.40}{5.16}$ & $\frac{5.11}{5.31}$ & $\frac{11.96}{12.12}$ \\
\hline
\end{tabular}

ISPC Generalization of scientific results, 


\begin{tabular}{|c|c|c|c|c|}
\hline ISRA (India) & $=1.344$ & SIS (USA) & ICV (Poland) & $=6.630$ \\
\hline ISI (Dubai, UAE & $=0.829$ & РИНЦ $($ Russia $)=0.234$ & PIF (India) & $=1.940$ \\
\hline GIF (Australia) & $=0.564$ & ESJI $($ KZ) $=1.042$ & IBI (India) & $=4.260$ \\
\hline IF & $=1.500$ & & & \\
\hline
\end{tabular}

\begin{tabular}{|c|c|c|c|c|c|c|c|c|c|c|c|}
\hline V & $\mathrm{COOC}_{2} \mathrm{H}_{5}$ & $\mathrm{CH}_{3}$ & $\mathrm{Ad}_{1}$ & $\mathrm{~S}$ & 91,6 & $\begin{array}{l}167- \\
168\end{array}$ & $\mathrm{C}_{27} \mathrm{H}_{29} \mathrm{~N}_{2} \mathrm{O}_{4} \mathrm{~S}_{2}$ & $\frac{63.81}{63.63}$ & $\frac{5.92}{5.74}$ & $\frac{5.38}{5.50}$ & $\frac{12.36}{12.5}$ \\
\hline VI & $\mathrm{CONH}_{2}$ & $\mathrm{CH}_{3}$ & $\mathrm{Ad}_{1}$ & $\mathrm{Se}$ & 77,8 & $\begin{array}{l}308- \\
309.5\end{array}$ & $\mathrm{C}_{25} \mathrm{H}_{26} \mathrm{~N}_{3} \mathrm{O}_{3} \mathrm{SSe}$ & $\frac{57.29}{56.92}$ & $\frac{5.19}{4.97}$ & $\frac{7.76}{7.97}$ & --- \\
\hline VII & $\mathrm{COOC}_{2} \mathrm{H}_{5}$ & $\mathrm{CH}_{3}$ & $\mathrm{CH}_{3}$ & S & --- & $\begin{array}{l}168- \\
169 \\
\end{array}$ & $\mathrm{C}_{18} \mathrm{H}_{18} \mathrm{~N}_{2} \mathrm{O}_{4} \mathrm{~S}_{2}$ & $\frac{56.11}{55.37}$ & $\frac{4.36}{4.65}$ & $\frac{7.41}{7.17}$ & $\frac{16.29}{16.4}$ \\
\hline VIII & $\mathrm{COOC}_{2} \mathrm{H}_{5}$ & $\mathrm{H}$ & $\mathrm{CH}_{3}$ & $\mathrm{~S}$ & --- & $\begin{array}{l}178- \\
179\end{array}$ & $\mathrm{C}_{17} \mathrm{H}_{16} \mathrm{~N}_{2} \mathrm{O}_{4} \mathrm{~S}_{2}$ & $\frac{54.61}{54.24}$ & $\frac{4.44}{4.28}$ & $\frac{7.09}{7.44}$ & $\frac{16.88}{17.05}$ \\
\hline
\end{tabular}

Data of PMR- and IR-spectra.

\begin{tabular}{|c|c|c|c|c|c|c|c|c|c|}
\hline № & \multicolumn{6}{|c|}{ PMR-spectra, $\delta$, m.g. } & \multicolumn{3}{|c|}{ IR-spectra, $v, \mathrm{~cm}^{-1}$} \\
\hline Compound & $\mathrm{CH}_{3}$ & $\mathrm{CH}_{2}$ & $\mathrm{NH}$ или $\mathrm{NH}_{2}$ & $\mathrm{Ad}_{1}$ & Arom. & Pyridyl & $\mathrm{NH}$ & $\mathrm{SO}_{2}$ & $\mathrm{C}=\mathrm{O}$ \\
\hline I & 1.95 & $\begin{array}{c}2.05 \\
\text { Acetone- } \\
\mathrm{D}_{6}\end{array}$ & $\begin{array}{c}5.6 \\
\text { Acetone- } \\
\mathrm{D}_{6}\end{array}$ & 2.55 & $\begin{array}{l}7.35- \\
7.75\end{array}$ & 7.65 & 3400 & $\begin{array}{l}1455 \\
1130\end{array}$ & --- \\
\hline II & $\begin{array}{l}1.30 \\
1.80\end{array}$ & $\begin{array}{c}2.05 \\
\text { Acetone- } \\
\mathrm{D}_{6}\end{array}$ & $\begin{array}{c}6.85 \\
\text { Acetone- } \\
\mathrm{D}_{6}\end{array}$ & 2.10 & $\begin{array}{l}7.4- \\
7.45\end{array}$ & 7.65 & 3440 & $\begin{array}{l}1450 \\
1160\end{array}$ & 1695 \\
\hline III & --- & $\begin{array}{c}8.2 \\
\text { DMSO- } \\
\mathrm{D}_{6}\end{array}$ & & & & & 3395 & $\begin{array}{l}1455 \\
1170\end{array}$ & 1695 \\
\hline IV & --- & +-- & --- & --- & --- & --- & 3310 & $\begin{array}{l}1455 \\
1165\end{array}$ & 1685 \\
\hline $\mathrm{V}$ & 1.45 & DMSO & $\begin{array}{c}7,0 \\
\mathrm{CONH}_{2} \\
8,25\end{array}$ & 2,50 & $7,3-7,6$ & 7,7 & $\begin{array}{c}3400 \\
\mathrm{CONH}_{2} ; \\
3320\end{array}$ & $\begin{array}{l}1450 \\
1145\end{array}$ & 1650 \\
\hline VI & --- & --- & --- & --- & --- & --- & 3430 & $\begin{array}{l}1490 \\
1160\end{array}$ & 1720 \\
\hline
\end{tabular}

\section{References:}

1. Chang Ming P, Ruju BC (2003) Patent USA 6541492, application. 27.12.2003. Published. 01.04 .2003

2. Polniazek RO, Wang X, Debal tetreys, Pandit CR (2003) Patent USA 6515130, application. 24.08.1998. Published 04.02.2003

3. Tarisuka K, Okasi M, Yamomoto N, Misibisu Seysi K (2008) application 63-44534(Japan), application. 11.08.2006. K-Ni 61-189020. Published 23.02.2008

4. Tosheva M, Antonova A (2005) Sofia University, chemistry department. 2005, 91, p.149-152,

5. Braje WM, Haupt A, Labirch W, Grandel R, Darye R, Turner S (2008) Patent USA 7320979, application 13.04.2004. Published 22.01.2008

6. Novikov MV, Mikhailov AM, Konishim ME, Vasilyuk MV, Kotegov VP, Vakhrin MI (2009)
Patent Russia. 2364594. application 09.01.2008. Published 20.08.2009

7. Zhong Zhimli, Chen Rong, Xing Ronga, Chen Xiaolin, Lui Song, Guo Zhanyong Zi Xia, Wang Lin, Li Pengcheng (2007) karbohydr Res., 2007, 342, №16. p.2390-2395

8. Mohammed MJ (2007) Bulg. Chem. Commun, 2007, 39, №2. p.152-158. РЖX.08.23-19O.109

9. Litvinov VP, Apenova EE, Sharanin YA, Shestopalov AM (1985) Synthesis of 6-(1adamantyl)-3-cyanopyridine1 $\mathrm{H}$ )-one and selenone. Journal of Orgn. Chem. 1985. -21, № 3, p. 669-670.

10. Litvinov VP, Apenova EE, Sharanin YA, Shestopalov AM (1984) Synthesis of 6(adamantyl-1)-3-cyano-2(1H)-pyridintion.

Russian Chemical Bulletin. Division of Chemical Sciences-1984. №10, p. 2408. 